\title{
Influence de la durée de conservation sous réfrigération du lait cru sur la conservabilité du lait U.H.T.
}

\author{
par \\ J. MOTTAR*
}

\section{R é s u m é}

L'auteur a étudié l'influence de la durée de conservation sous réfrigération du lait cru sur la conservabilité du lait UHT. Cette influence se ramène principalement à un effet d'enzymes protéolytiques thermorésistantes. La présence d'enzymes de ce genre est déterminée dans une importante mesure par la qualité bactériologique du lait cru. Le temps pendant lequel la matière première peut être stockée, même sous réfrigération, est limité pour cette raison.

Les résultats donnent à supposer que lorsqu'on fabrique du lait UHT à partir d'un lait cru de bonne qualité bactériologique, c'est-àdire à moins d'environ $10^{4}$ bactéries au $\mathrm{ml}$ lors de la traite, ce lait cru peut être stocké sous réfrigération pendant un maximum de $72 \mathrm{~h}$. Cette durée de stockage maximale est réduite à environ $48 \mathrm{~h}$ si le lait contient plus de $10^{5}$ bactéries au $\mathrm{ml}$. Un stockage de plus longue durée implique le risque de formation de protéases bactériennes thermorésistantes, ce qui limite dans une mesure significative la conservabilité du lait UHT. Des phénomènes de protéolyses peuvent cependant se manifester aussi dans du lait UHT fabriqué à partir d'un lait cru stocké moins longtemps. Ces phénomènes-là sont imputables à l'activité résiduelle du système protéolytique propre du lait.

Mots clés : Conservation du lait cru - Lait U.H.T. - Conservabilité - Protéase du lait - Protéases microbiennes.

* Station Laitière de l'Etat Melle. Centre de Recherches Agronomiques de l'Etat, Gand (Belgique). 


\section{S u m m a r y \\ INFLUENCE OF RAW MILK STORAGE \\ ON THE KEEPING QUALITY OF UHT MILK}

The influence of raw milk storage on the shelf life of UHT milk was investigated. Such influence is mainly reduced to the effect of thermoresistant proteolytic enzymes. The presence of such enzymes is greatly determined by the bacteriological quality of the raw milk. This results in a limited storage time of the raw milk, even under deep-cooled conditions.

When UHT milk is obtained from milk of good bacteriological quality, i.e. initially containing less than approx. $10^{4}$ bacteria per $\mathrm{ml}$, the results have shown that the maximum storage time at deepcooled temperatures is, as a rule, $72 \mathrm{~h}$. If the initial raw milk contains more than $10^{5}$ bacteria per $m l$, the maximum keeping time is approx. $48 \mathrm{~h}$. Longer storage times may result in the formation of thermoresistant bacterial proteases, which significantly limit the shelf life of the UHT milk obtained. Proteolytic phenomena can, however, also occur in UHT milk obtained from raw milk stored for shorter periods. These may be ascribed to a residual activity of the natural proteolytic system.

Key words: Raw milk storage - U.H.T. milk - Shelf life - Milk protease Bacterial proteases.

\section{INTRODUCTION}

La conservation par réfrigération du lait cru est désormais généralisée. Il arrive fréquemment que le lait soit stocké pendant $48 \mathrm{~h}$ et plus à une température de 4 à $6^{\circ} \mathrm{C}$. On sait que des micro-organismes psychrotrophes peuvent encore se développer à des températures aussi basses et que certains d'entre eux sont responsables de la formation d'enzymes thermorésistantes. La conservabilité du lait traité à ultra haute température est déterminée dans une large mesure par la présence de protéases thermostables (Adams et coll,, 1975 ; Ged et Alais, 1976; Mottar et coll. 1979; Richardson et Newstead, 1979 ; Driessen, 1981).

Nous avons recherché en quelle mesure l'évolution de la matière première stockée sous réfrigération influe sur la conservabilité du lait U.H.T. La qualité du lait cru a été étudiée des points de vue microbiologique et enzymatique et mise en corrélation avec le comportement organoleptique pendant la conservation à $20^{\circ} \mathrm{C}$ du lait chauffé à ultra haute température et conditionné aseptiquement. Nous avons tenté d'évaluer l'importance du rôle du système protéolytique propre du lait, ainsi que celle de l'influence des protèses thermorésistantes d'origine bactérienne. 


\section{MATERIEL ET METHODES}

\section{II.1. Conditions de traitement}

Du lait de mélange cru, qui avait présenté au moment de la traite des degrés de contamination variables, a été soumis, après différentes périodes de stockage à $4-6^{\circ} \mathrm{C}$, à un traitement U.H.T. dans une installation A.P.V. Junior Twin. Le lait était préchauffé à $75^{\circ} \mathrm{C}$ et ensuite porté à $142-144^{\circ} \mathrm{C}$ par injection de vapeur; le temps de séjour était de 5 secondes. Le lait ainsi chauffé à ultra haute température a été conditionné aseptiquement dans des bouteilles de polyéthylène de 0,5 litre préalablement stérilisées, puis conservé 4 mois à $20^{\circ} \mathrm{C}$ et dans l'obscurité.

Selon leur qualité bactériologique initiale, c'est-à-dire au moment de la traite, les matières premières pouvaient être réparties en 2 groupes, l'un à nombre de germes total inférieur à $10^{4} / \mathrm{ml}$, l'autre à nombre de germes total supérieur à $10^{5} / \mathrm{ml}$. Le premier groupe a fait l'objet de 4 répétitions, le second de 3 .

\section{II.2. Déterminations bactériologiques}

Le nombre total de germes a été déterminé sur Plate Count Agar. Le temps d'incubation fut de 3 jours à $30^{\circ} \mathrm{C}$. Les boîtes de Petri destinées au comptage des psychrotrophes ont été incubées 10 jours à $6,5^{\circ} \mathrm{C}$.

Les micro-organismes protéolytiques ont été dénombrés sur un milieu de caséinate (Speck, 1976).

\section{II.3. Analyses chimiques}

L'activité protéasique a été déterminée par incubation anaérobique du lait à $37^{\circ} \mathrm{C}$ en présence de $0,01 \%$ de thimerosal. La quantité d'azote non caséique (N.C.N.) a été déterminée après $0,3,9$ et 12 jours. Cette activité a été calculée à l'aide d'une régression linéaire simple et exprimée en augmentation de N.C.N. par unité de temps $(\mathrm{dNCN} / \mathrm{dt}$, mg par $24 \mathrm{~h}$ et pour $100 \mathrm{~g}$ de lait).

La protéolyse dans le lait U.H.T. a été suivie par des déterminations mensuelles de la quantité d'azote non caséique (N.C.N.) et non protéique (N.P.N.) au cours de la conservation sans réfrigération. Le degré de protéolyse a été déterminé comme augmentation des différents paramètres par mois et pour $100 \mathrm{~g}$ de lait, calculé à l'aide d'une analyse de régression linéaire simple.

Les fractions N.C.N. et N.P.N. ont été isolées selon la méthode d'Aschaffenburg et Drewry (1959) et l'azote a été dosé par le procédé micro-Kjeldahl. 
La détermination de l'acide sialique a été effectuée conformément à la directive L 213 de la C.E.E. (1981). Les glycomacropeptides, dont l'acide sialique, sont précipités à l'acide phosphotungstique en partant d'un filtrat à $12 \%$ d'acide trichloracétique. L'acide sialique, libéré par hydrolyse acide, forme avec le résorcinol un complexe coloré qui peut être déterminé par spectrophotométrie à $580 \mathrm{~nm}$.

La caséine précipitée par voie isoélectrique, lavée et lyophylisée a été séparée qualitativement par électrophorèse sur gel d'amidon (Mottar, 1983). Les différentes fractions ont été quantifiées par densitométrie à $600 \mathrm{~nm}$ à l'aide d'un densitomètre Beckman CDS-200. Les différentes fractions de caséine ont pu être calculées en $\mathrm{mg} / 100 \mathrm{~g}$ de lait d'après le pourcentage de répartition et la teneur en caséine.

\section{II.4. Appréciation du goût}

Le goût du lait U.H.T. a été apprécié après 3 mois de conservation à $20^{\circ} \mathrm{C}$ par un test de tétrades modifié, tel que décrit par Mottar et coll. (1979).

\section{RESULTATS ET DISCUSSION}

\section{III.1. Développement microbien au cours de la conservation sous réfrigération du lait cru}

La figure 1 donne un aperçu du développement de la population microbienne au cours du stockage sous réfrigération du lait cru, pour 2 groupes de lait présentant des niveaux de contamination initiale différents.

Dans le premier groupe de matières premières, le lait était initialement de très bonne qualité bactériologique : le nombre de germes total était inférieur à $10^{4} / \mathrm{ml}$. Dans le $2^{\mathrm{e}}$ groupe, le nombre de germes total présents dans le lait, stocké sous réfrigération depuis moins de $24 \mathrm{~h}$, était supérieur à $10^{5} / \mathrm{ml}\left(1,3-2,7.10^{5}\right)$ de sorte que ce lait pouvait être considéré comme de moins bonne qualité bactériologique.

Comme on pouvait s'y attendre, le nombre de germes a augmenté pendant le stockage à $4-6^{\circ} \mathrm{C}$. On peut dire qu'en général le nombre de bactéries du lait s'élève d'autant plus haut pendant le stockage sous réfrigération que le nombre de germes initial est élevé (Busse, 1965 ; Keller, 1968). Ceci s'explique par le fait que le nombre de germes en phase de croissance active est généralement plus important dans un lait à nombre de germes élevé, comparativement à un lait à petits nombres de bactéries (Flückiger, 1976).

Dans le lait cru qui était de bonne qualité bactériologique aussitôt après la traite, le nombre de germes total a augmenté, en 


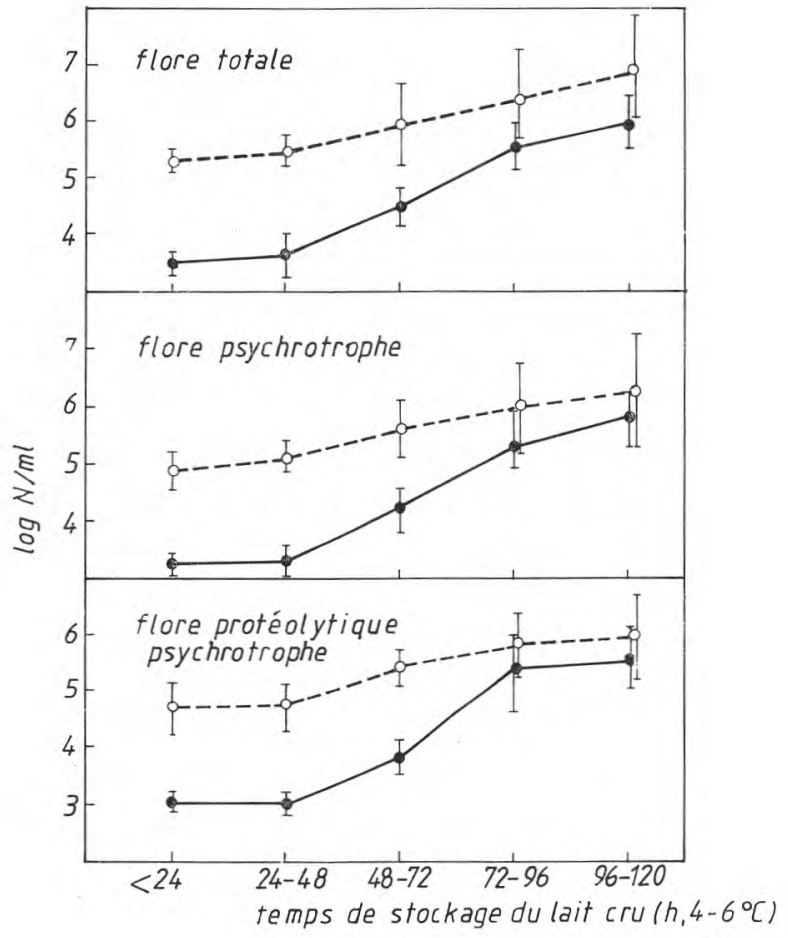

fig. 1

Développement microbien dans le lait cru au cours du stockage sous réfrigération (-) : moyennes des matières premières contenant initialement $<10^{4}$ bactéries au $\mathrm{ml} ; 0--0$ : moyennes des matières premières contenant initialement $>10^{5}$ bactéries au $\mathrm{ml} ; \Phi$ : valeurs extrêmes).

Microbial development during the cooled storage of raw milk. (•- $\bullet$ mean values for raw milks containing initially $<10^{4}$ micro-organisms per $\mathrm{ml}_{i}$ 0--o: mean values for raw milks containing initially $>10^{5}$ micro-organisms per $\mathrm{ml}$; $\Phi$ : extrem values).

moyenne, d'initialement $3.10^{3}$ à $875.10^{3} \mathrm{ml}$ après $96 \mathrm{~h}$ de stockage au froid. Dans le lait obtenu dans de moins bonnes conditions d'hygiène, ce nombre a augmenté, en moyenne, de $2.10^{5}$ à $8.10^{6} / \mathrm{ml}$.

Le stockage sous réfrigération du lait entraînait une augmentation de la proportion de bactéries psychrotrophes. Le nombre de germes psychrotrophes initial était, en moyenne, de $45 \%$ du nombre de germes total. Après $96 \mathrm{~h}$ de stockage à $4-6^{\circ} \mathrm{C}$, la proportion atteignait $72,6 \%$. 


\section{III.2. Activités protéolytiques résiduelles dans le lait U.H.T., en fonction du temps de stockage du lait cru}

Les activités protéolytiques observées dans le lait cru de départ et dans le lait U.H.T. correspondant sont représentées sur la figure 2.

En ce qui concerne le lait cru de départ contenant moins de $10^{4}$ bactéries au $\mathrm{ml}$, dont moins de $2.10^{3}$ psychrotrophes, on peut admettre que les activités protéoloytiques observées, aussi bien dans ce lait cru que dans le lait U.H.T. correspondant, sont imputables à l'action du système protéolytique propre du lait.

Quand une matière première de ce genre, initialement de très bonne qualité bactériologique, était stockée sous réfrigération pendant plus de $72 \mathrm{~h}$, on pouvait observer, en règle générale, une nette

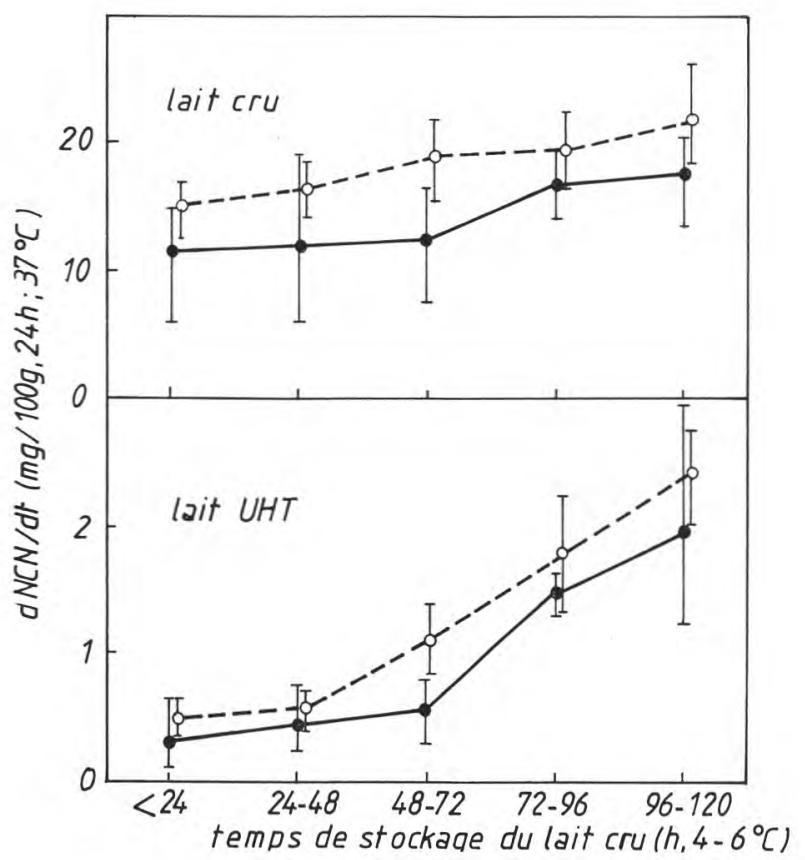

fig. 2

Activités protéolytiques dans le lait cru et dans le lait U.H.T en fonction de l'âge de la matière première $(\bullet-\bullet:$ moyennes des matières premières contenant initialement $<10^{4}$ bactéries au $\mathrm{ml} ; 0--0$ : moyennes des matières premières contenant initialement $>10^{5}$ bactéries au $\mathrm{ml} ; \Phi$ : valeurs extrêmes).

Proteolytic activities in raw and U.H.T.-milk as a function of storage time of the raw milk. (- : mean values for raw milks containing initially $<10^{4}$ microorganisms per ml; $0--0$ : mean values for raw milks containing initially $>10^{5}$ micro-organisms per $\mathrm{ml}$; $\Phi$ : extreme values). 
augmentation de l'activité protéasique ainsi que de l'activité résiduelle dans le lait U.H.T. correspondant.

Quant au lait cru qui était déjà initialement de moins bonne qualité bactériologique, c'est-à-dire qui contenait plus de $10^{5}$ bactéries au $\mathrm{ml}$, une augmentation d'activité analogue fut déjà observée après $48 \mathrm{~h}$ de stockage sous réfrigération.

L'augmentation d'activité protéolytique allait de pair avec un développement notable de la flore psychrotrophe (fig. 1). Ceci donne à supposer que des quantités mesurables de protéases bactériennes thermarésistantes sont formées après $72 \mathrm{~h}$ de stockage du lait cru ou même déjà après $48 \mathrm{~h}$, selon l'état bactériologique initial de la matière première.

Il convient de remarquer que ce n'est pas le nombre de germes total tel quel, qui est significatif pour la présence d'enzymes protéolytiques thermostables, mais plutôt le nombre de germes psychrotrophes et, plus précisément, la flore protéolytique psychrotrophe (Mottar, 1981). Il est admis en général que plus le nombre de germes total du lait de mélange cru stocké sous réfrigération est élevé, plus le nombre de psychrotrophes est important (Bossuyt, 1976). Ces données le confirment.

Peu d'opinions, du reste très divergentes, ont été émises quant au nombre de bactéries psychrotrophes nécessaire pour détecter des protéases thermostables. Adams et coll. (1975) ont relevé des quantités mesurables d'enzymes à partir d'environ $10^{4}$ germes psychrotrophes par $\mathrm{ml}$, avec des conséquences néfastes pour la conservabilité du lait U.H.T. Gebre-Egziabher et coll. (1980) ont constaté une protéolyse à partir de teneurs de $27.10^{3}$ psychrotrophes par ml. Law (1979) affirme cependant qu'il faut plus de $10^{7}$ psychrotrophes par $\mathrm{ml}$ pour susciter ce phénomène. Il peut être déduit de nos résultats représentés sur les figures 1 et 2 , que des protéases bactériennes peuvent être détectées, par la méthode que nous avons appliquée, à partir d'en moyenne $25.10^{4}$ bactéries psychrotrophes protéolytiques par $\mathrm{ml}$. Cette moyenne a pu être calculée en partant des nombres de bactéries psychrotrophes protéolytiques présentes, d'une part, dans les matières premières qui contenaient initialement moins de $10^{4}$ bactéries au $\mathrm{ml}$ et avaient été stockées 72 à $96 \mathrm{~h}$ à $4-6^{\circ} \mathrm{C}$ et, d'autre part, dans le lait contenant initialement plus de $10^{5}$ bactéries au $\mathrm{ml}$ et stocké au froid pendant 96 à $120 \mathrm{~h}$.

Les différences d'opinion s'expliquent peut-être par l'emploi de méthodes de détection différentes et à sensibilités différentes, dans lesquelles l'enzyme pouvait agir sur le substrat pendant des périodes d'incubation très différentes. En outre, le stade de croissance de la population bactérienne joue certainement aussi un rôle.

On peut aussi déduire des données de la figure 2 que la protéase naturelle du lait est douée d'une certaine thermorésistance, qui lui permet de survivre en partie à un traitement U.H.T. modéré $\left(142-144^{\circ} \mathrm{C} 5 / \mathrm{s}\right.$; 
procédé direct). Ces observations concordent avec les constatations de Björck (1972), Ged et Alais (1975), Snoeren et Evers (1978), Driessen et Van der Waals (1978) et Snoeren et coll. (1979).

\section{III.3. Protéolyse dans le lait U.H.T. au cours de la conservation à $20^{\circ} \mathrm{C}$, en fonction du temps de stockage du lait cru}

La protéolyse dans le lait U.H.T. au cours de la conservation sans réfrigération a été déterminée en suivant la libération de produits typiques de la dégradation de protéines tels que l'azote non caséique (N.C.N.), l'azote non protéique (N.P.N.) et l'acide sialique. L'acide sialique se rencontre principalement dans la fraction stabilisatrice de la caséine (Marrier et coll., 1963) et en est libérée par certaines enzymes protéolytiques (Alais et Jolles, 1961 ; Jolles et coll., 1961; Nitschmann et Beeby, 1960).

L'évolution du N.C.N. et celles du N.P.N. et de l'acide sialique au cours de la conservation à $20^{\circ} \mathrm{C}$ du lait U.H.T., en fonction de l'âge de la matière première sont représentées respectivement sur les figures 3,4 et 5 .

Les résultats relatifs à la protéolyse concordent très bien avec ce que l'on pouvait prévoir d'après les activités résiduelles déterminées dans le lait U.H.T.

On peut déduire de ces données que dans le cas du lait de bonne qualité bactériologique initiale $\left(<10^{4}\right.$ bactéries au $\mathrm{ml}$ lors de la traite), il ne s'est généralement produit qu'une protéolyse restreinte au cours de la conservation sans réfrigération du lait U.H.T. fabriqué dans les $72 \mathrm{~h}$ suivant la traite. Considérant les faibles teneurs en germes du lait de départ, on peut admettre que des protéases bactériennes ne sont pas encore présentes ou ne sont présentes qu'en quantités négligeables dans la matière première, de sorte que l'activité protéolytique observée dans le lait U.H.T. doit être imputée à une activité résiduelle de la protéase naturelle du lait.

Le stockage pendant plus de $72 \mathrm{~h}$ d'un lait cru de ce genre a entraîné un net accroissement de la protéolyse dans le lait U.H.T. Ceci a pu être mis en corrélation avec le développement de micro-organismes psychrotrophes, plus précisément psychrotrophes protéolytiques dans le lait cru. Des quantités mesurables d'enzymes bactériennes thermorésistantes ont pu être détectées de ce fait après la période de stockage susdite.

Quand on partait de lait cru dont la qualité bactériologique était déjà moins bonne dès la traite (nombre de germes $>10^{5}$ par $\mathrm{ml}$ ), on pouvait déduire des données que déjà lorsque ce lait cru a été stocké $48 \mathrm{~h}$ sous réfrigération, une protéolyse nettement accrue peut se manifester dans le lait U.H.T. correspondant. II apparaît aussi que des protéases d'origine bactérienne peuvent déjà être présentes en quantités mesurables après 2 jours de stockage sous réfrigération de 


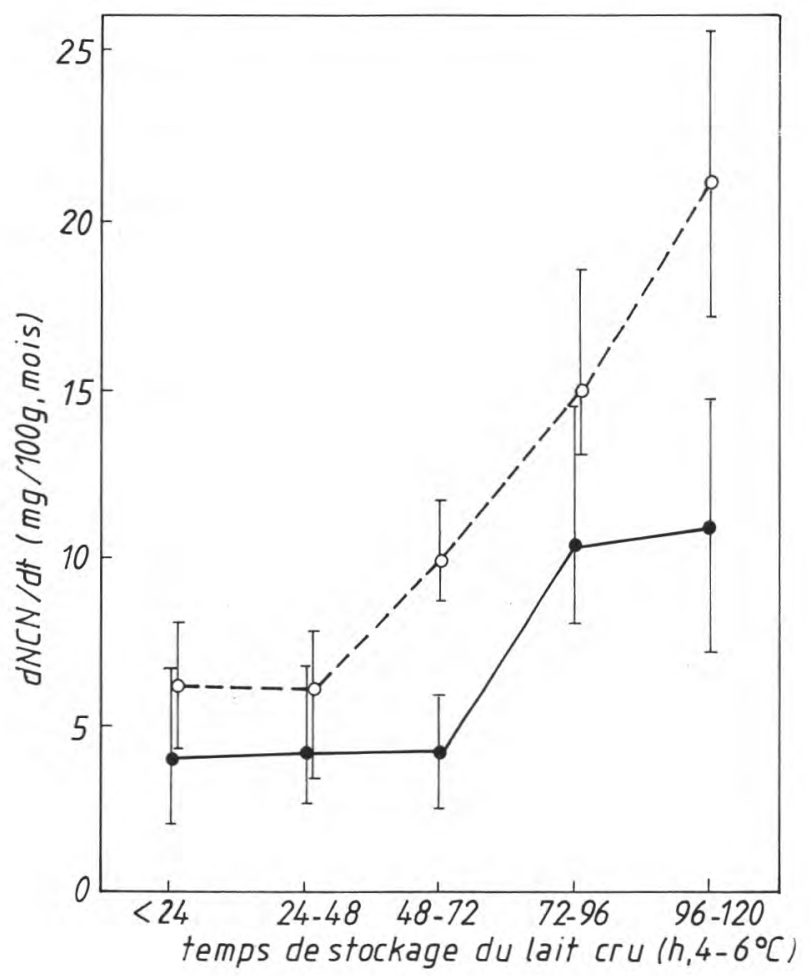

fig. 3

Evolution de la teneur en azote non-caséinique du lait U.H.T. pendant la conservation à $20^{\circ} \mathrm{C}$ en fonction de l'âge de la matière première $(-\bullet:$ moyennes des matières premières contenant initialement $<10^{4}$ bactéries au $\mathrm{ml} ; 0-0$ : moyennes des matières premières contenant initialement $>10^{5}$ bactéries au $\mathrm{ml}$; $\Phi$ : valeurs extrêmes).

Evolution of the non-casein nitrogen content in U.H.T. milk during storage at $20^{\circ} \mathrm{C}$ as a function of the storage time of the raw milk. (- - : mean values for raw milks containing initially $<10^{4}$ micro-organisms per $\mathrm{ml}$; 0--0: mean values for raw milks containing initially $>105$ micro-organisms per $\mathrm{ml}_{\text {; }}$ 夲: extreme values).

la matière première. Ceci allait également de pair avec une augmentation appréciable du nombre de micro-organismes psychrotrophes dans le lait de départ (fig. 1).

L'augmentation soudaine de la teneur en acide sialique, observé conjointement avec le développement de micro-organismes protéolytiques psychrotrophes, donne à supposer que les protéases bactériennes et thermostables dégradent dans une plus large mesure la fraction de K-caséine, ce que ne fait pas la protéase naturelle du lait. 


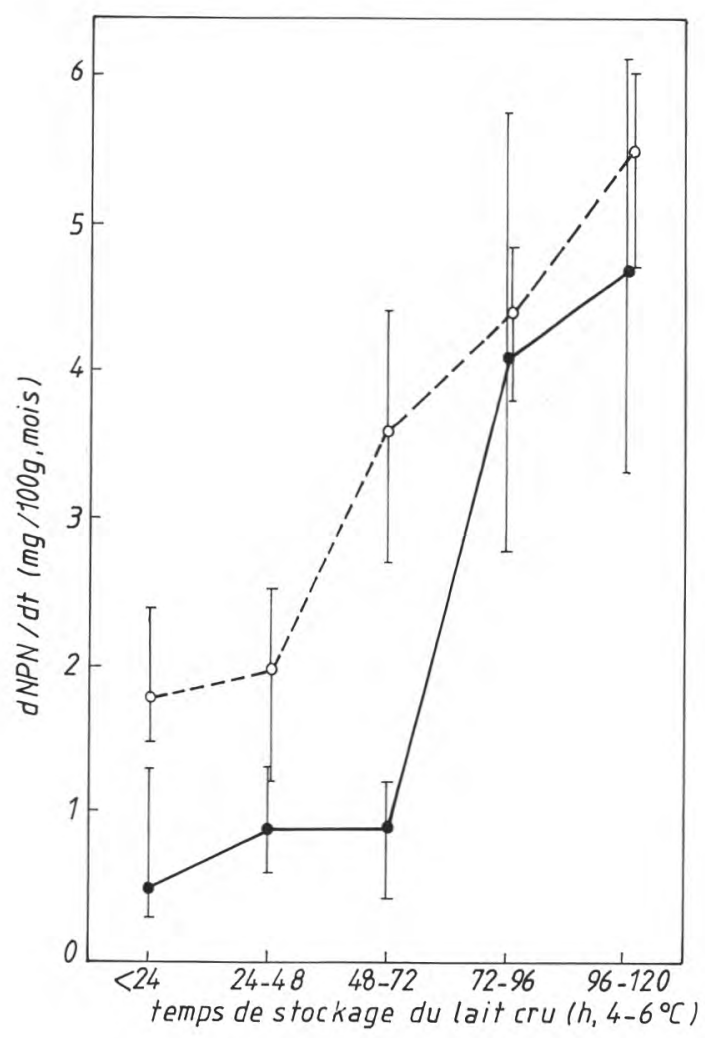

fig. 4

Evolution de l'azote non-protéinique du lait U.H.T. pendant la conservation à $20^{\circ} \mathrm{C}$ en fonction de l'âge de la matière première $(\bullet \bullet:$ : moyennes des matières premières contenant initialement $<10^{4}$ bactéries au $\mathrm{ml} ; 0--0$ : moyennes des matières premières contenant initialement $>10^{5}$ bactéries au ml; $\Phi$ : valeurs extrêmes).

Evolution of the non-protein nitrogen content in UHT milk during storage at $20^{\circ} \mathrm{C}$ as a function of the storage time of the raw milk. ( - $\bullet$ : mean values for raw milks containig initially $<10^{4}$ micro-organisms per $\mathrm{ml} ; 0-0$ : mean values for raw milks containing initially $>10^{5}$ micro-organisms per $\mathrm{ml}$; $\Phi$ : extreme values).

Le système protéolytique propre du lait dégrade cependant aussi la K-caséine, fut-ce dans une moindre mesure, ce qui correspond aux observations de Yamauchi et Kaminogawa (1972), lesquels sont arrivés à la conclusion que la para-K-caséine est aussi formée sous l'action de la protéase du lait et non seulement par les protéases bactériennes. Chen et Ledford (1972) prétendent que la K-caséine n'est pas dégradée par la protéase propre du lait. 


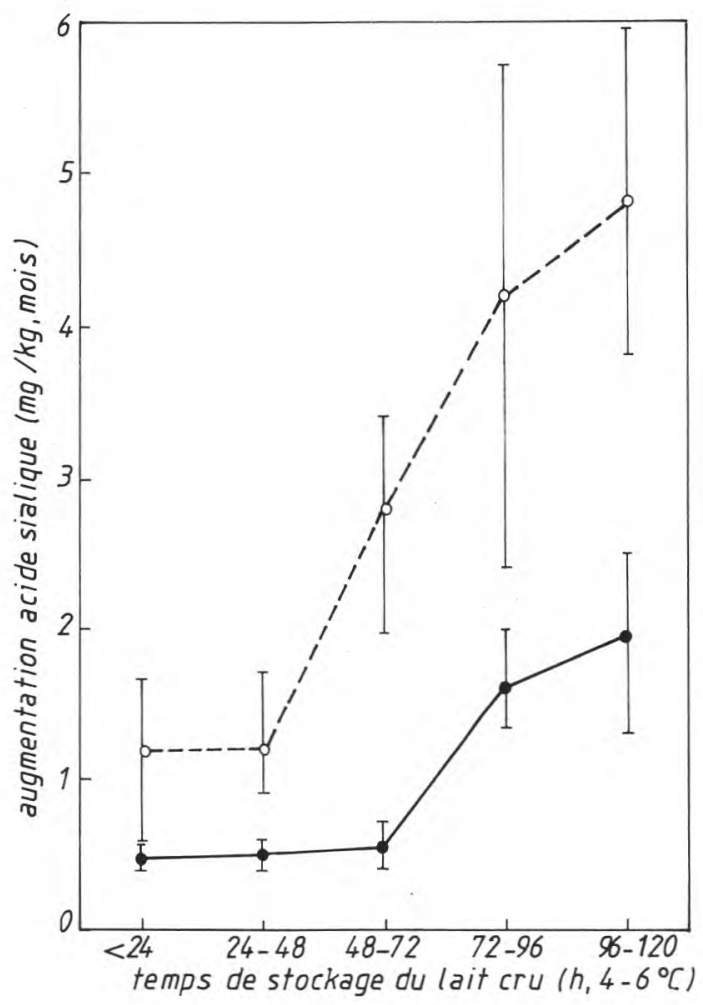

fig. 5

Evolution d'acide sialique du lait U.H.T. pendant la conservation à $20 \mathrm{C}$ en fonction de l'âge de la matière première (- - : moyennes des matières premières contenant initialement $<10^{4}$ bactéries au $\mathrm{ml}$; $0--0$ : moyennes des matières premières contenant initialement $>10^{5}$ bactéries au $\mathrm{ml} ; \Phi$ : valeurs extrêmes).

Evolution of the sialic acid content in U.H.T. milk during storage at $20^{\circ} \mathrm{C}$ as a function of the storage time of the raw milk. (- $\bullet$ : mean values for raw milks containing initially $<10^{4}$ micro-organisms per $\mathrm{ml}$; $0--0$ : mean values for raw milks containing initially $>10^{5}$ micro-organisms per $\mathrm{ml}$; $\Phi$ extreme values).

Les résultats permettent de supposer que, sous l'influence du système protéolytique propre du lait, la caséine est dégradée surtout en fragments plus petits, dont la nature est toutefois encore principalement protéinique.

Dans le cas du lait U.H.T. fabriqué à partir d'une matière première à nombre de germes peu élevé et dont on peut supposer que l'activité enzymatique bactérienne était faible, on peut effectivement calculer que la part de la fraction N.P.N. dans l'augmentation du 


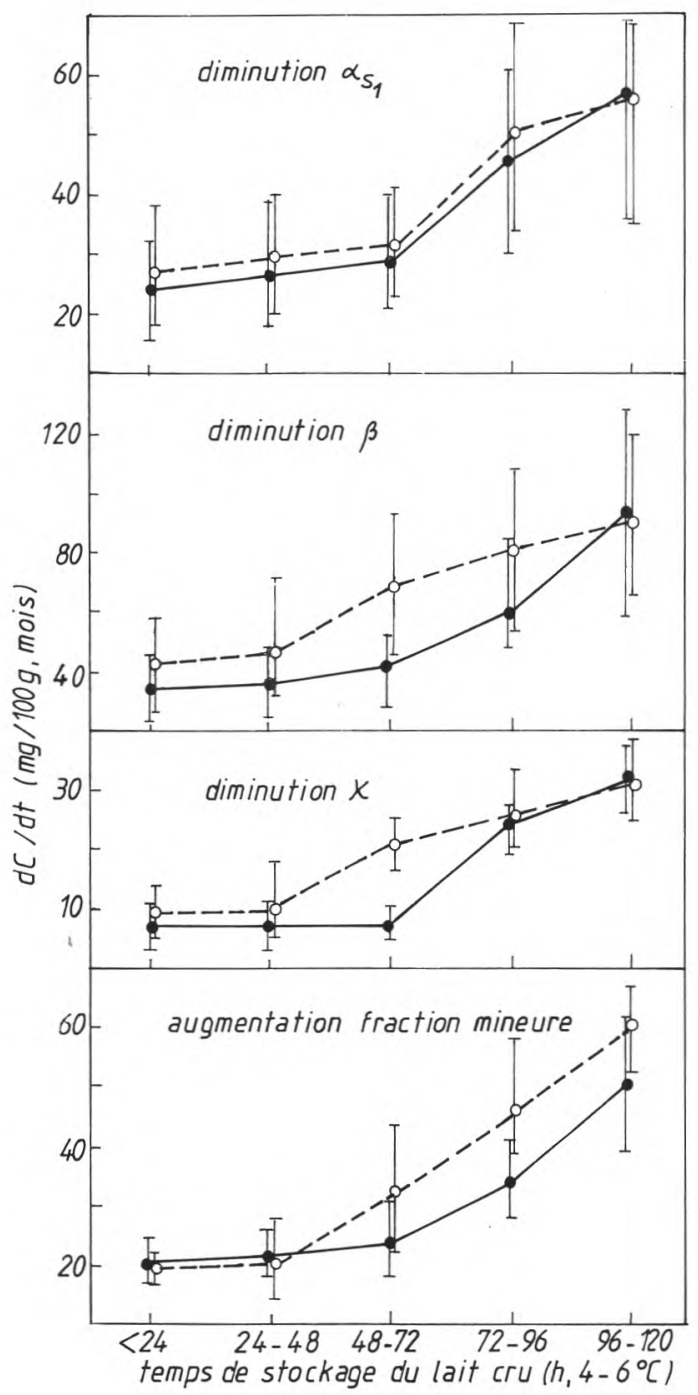

fig. 6

Dégradation de la caséine du lait U.H.T. pendant la conservation à $20^{\circ} \mathrm{C}$ en fonction de l'âge de la matière première (- $\bullet$ : moyennes des matières premières contenant initialement $<10^{4}$ bactéries au $\mathrm{ml} ; 0--0$ : moyennes des matières premières contenant initialement $>10^{5}$ bactéries au $\mathrm{ml} ; \Phi$ : valeurs extrêmes.

Casein degradation in U.H.T. milk during storage at $20^{\circ} \mathrm{C}$ as a function of the storage time of the raw milk (- mean values for raw milks containing initially $<10^{4}$ micro-organisms per $\mathrm{ml}$; 0 --0: mean values for raw milks containing initially $>105$. micro-organisms per $m l ; \Phi$ extrem values). 
N.C.N. ne s'élevait, en moyenne, qu'à $28 \%$. Par contre, dans le cas du lait U.H.T. fabriqué à partir d'une matière première à nombre de germes élevé et dont on peut admettre qu'elle contenait réellement des protéases bactériennes thermorésistantes, la part du N.P.N. dans l'augmentation du N.C.N. atteignait, en moyenne, $42 \%$. Ceci permet de supposer que la dégradation de la caséine par les protéases thermorésistantes d'origine bactérienne est plus poussée que la dégradation par le système protéolytique propre du lait et produit par conséquent plus de substances azotées dont la nature n'est plus protéinique. Ces observations confirment les suppositions de Driessen (1981).

La protéolyse a également été suivie par électrophorèse. Les résultats des différentes séries d'essais sont reproduits dans la figure 6, qui donne aussi un aperçu du profil de dégradation de la caséine au cours de la conservation du lait U.H.T. à $20^{\circ} \mathrm{C}$, en partant de matières premières de qualités bactériologiques diverses, après diverses périodes de stockage à 4-6 $\mathrm{C}$.

De nettes différences ont été observées dans le profil de dégradation de la caséine selon l'âge et la qualité bactériologique du lait cru. Si, comme on peut supposer d'après ce qui précède, l'on admet que des effets de protéases bactériennes peuvent être observés dans le lait U.H.T. fabriqué à partir de lait cru de bonne qualité bactériologique initiale et stocké au moins 72 h sous réfrigération, ainsi que dans le lait U.H.T. fabriqué à partir de lait cru de moins bonne qualité initiale et stocké au moins $48 \mathrm{~h}$ sous réfrigération, on peut déduire des données électrophorétiques :

- que le système protéolytique propre du lait dégrade principalement la $\beta$-caséine et, dans une moindre mesure, la caséine $\alpha s_{1}$. Ceci concorde avec les constatations de Noomen (1975) et de Yamauchi et Kaminogawa (1972). La K-caséine est aussi dégradée, quoique dans une mesure limitée, comme également observé par Yamauchi et Kaminogawa (1972) et déjà démontré par la libération d'acide sialique. De ces réactions enzymatiques résultent des produits de dégradation spécifiques;

- que les protéases bactériennes thermorésistantes dégradent nettement les caséines $\beta$ et $\mathrm{K}$ et dans une moindre mesure la caséine $\alpha \mathrm{s}_{1}$. Ceci est à mettre en concordance avec les observations de Law (1977; 1979) et de Richardson et Te Whaiti (1978).

\section{III.4. Evolution du goût et de la stabilité du lait U.H.T. pendant la conservation à $20^{\circ} \mathrm{C}$ en fonction du temps de stockage du lait cru}

Les pertes de goût observées pour les différentes fabrications de lait U.H.T. sont indiquées dans la figure 7. L'évolution gustative concorde très bien avec ce qu'on pouvait prévoir d'après les processus protéolytiques observés. Pour le lait U.H.T. fabriqué dans les 


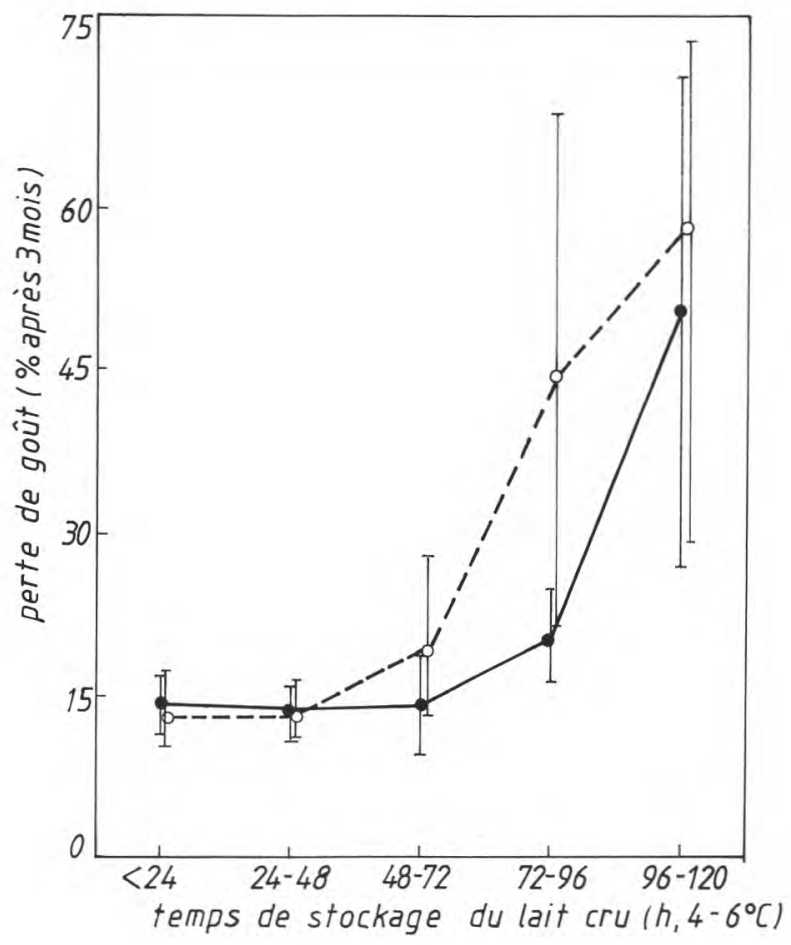

fig. 7

L'évolution gustative du lait U.H.T. pendant la conservation à $20^{\circ} \mathrm{C}$ en fonction de l'âge de la matière première (- - : moyennes des matières premières contenant initialement $<10^{4}$ bactéries au $\mathrm{ml}$; $\mathrm{O}-\mathrm{-} \mathrm{O}$ : moyennes des matières premières contenant initialement $>10^{5}$ bactéries au $\mathrm{ml} ; \Phi$ : valeurs extrêmes).

Evolution of the taste of U.H.T. milk during storage at $20^{\circ} \mathrm{C}$ as a function of the storage time of the raw milk (- mean values for raw milks containing initially $<10^{4}$ micro-organism per $\mathrm{ml}$; $\mathrm{O}-\mathrm{-O}$ : mean values for raw milks containing initially $>105$ micro-organisms per $\mathrm{ml}$; $\Phi$ : extreme values).

$72 \mathrm{~h}$ suivant la traite de lait à faible nombre de germes initial $\left(<10^{4}\right.$ au $\mathrm{ml}$ ) ou fabriqué dans les $48 \mathrm{~h}$ suivant la traite de lait à nombre de germes initial relativement élevé $\left(>10^{5}\right.$ au $\mathrm{ml}$ ) les pertes de goût enregistrées pendant la conservation étaient analogues et, en règle générale, plutôt faibles. Celles-ci doivent être imputées à la faible action résiduelle de la protéase naturelle du lait. Il est apparu, par contre, que le lait U.H.T. fabriqué à partir de lait cru stocké plus longtemps sous réfrigération subissait en général des pertes de goût plus considérables, imputables surtout à l'apparition d'un goût amer. Les anomalies de goût du lait U.H.T. fabriqué à partir de matières premières d'un certain âge peuvent être imputées dans une large 
mesure à l'action résiduelle des protéases bactériennes thermorésistantes.

Une gélification suivie d'une déstabilisation s'est aussi produite parfois dans du lait U.H.T. fabriqué à partir de lait cru stocké plusieurs jours sous réfrigération. Puisque le phénomène de la coagulation des protéines allait de pair avec des activités relativement intenses de protéases résiduelles, résultant du développement de bactéries psychrotrophes, les données permettent de supposer qu'une gélification est principalement une conséquence de processus enzymatiques.

\section{CONCLUSION}

La présence d'enzymes bactériennes thermorésistantes dans le lait est déterminée dans une importante mesure par l'âge de la matière première. Le lait cru ne peut être stocké, même sous réfrigération, que pendant un temps limité si l'on veut assurer au lait U.H.T. une conservabilité suffisante. Il est apparu que la durée de conservation dépend de la condition bactériologique du lait lors de la traite.

Quoique le nombre de germes total, tel quel, ne soit pas significatif pour la présence d'enzymes protéolytiques thermorésistantes, alors que le sont les bactéries protéolytiques psychrotrophes, il est possible de fixer des limites au nombre de germes total; en effet les bactéries protéolytiques psychrotrophes du lait cru y représentent un pourcentage plus ou moins constant et en cas de stockage sous réfrigération le nombre de ces bactéries augmente d'autant plus rapidement que le nombre initial de germes est élevé.

Lorsque, dans la fabrication de lait U.H.T., on part d'un lait cru de bonne qualité bactériologique, c'est-à-dire à moins de $10^{4}$ bactéries au $\mathrm{ml}$ lors de la traite, ce lait cru peut, en règle générale, être stocké sous réfrigération pendant $72 \mathrm{~h}$. Si la matière première contient initialement plus de $10^{5}$ bactéries au $\mathrm{ml}$, la durée normale du stockage à 4-6 $6^{\circ} \mathrm{C}$ est, en moyenne, de $48 \mathrm{~h}$. Un stockage de plus longue durée entraîne le risque de concentrations de protéases bactériennes thermorésistantes suffisamment élevées pour limiter significativement la conservabilité du lait U.H.T.

Des phénomènes de protéolyse peuvent toutefois se manifester aussi dans du lait cru stocké moins longtemps. Ces phénomènes sont imputables à l'activité résiduelle du système protéolytique propre du lait. Cette enzyme naturelle du lait fait également preuve d'une certaine thermostabilité, moins grande toutefois que la résistance opposée à la chaleur par certaines protéases bactériennes.

Il convient de remarquer que des intensités de chauffage relativement faibles ont été appliquées au cours de nos essais. L'inac- 
tivation des enzymes augmente avec l'intensité du traitement thermique, mais la valeur organoleptique et nutritive du lait U.H.T. s'en trouve diminuée.

\section{Bibliographie}

Adams (D. M.), Barach (J. T.), SPeck (M. L.) (1975). - Heat resistant proteases produced in milk by psychrotrophic bacteria of dairy origin. Journal of Dairy Science, 58, 828-834.

Alais (C.), Jolles (P.) (1961). - Etude comparée des caséinoglycopeptides formés par action de la présure sur les caséines de vache, de brebis et de chèvre. II. Etude de la partie non peptidique. Biochimica et Biophysica Acta, 51, 315-319.

Ashaffenburg (R.), Drewry (J.) (1959). - New procedure for the routine determination of various non-casein proteins of milk. 15th International Dairy Congress 3, 1631-1635.

BJoRCK (L.) (1973). - Modifications of casein in ultra-high temperature treated milk during storage. Milchwissenschaft, 28, 291-294.

BossuYT (R.) (1976). - Aktiviteitsverslag. Werkgroep ter verbetering van de kwaliteit van rauwe melk.

Busse (M.) (1965). - Milchkühlung und Bakterienflora. Deutsche MolkereiZeitung, 86, 1777-1778.

CHEN (J. H.), LEDFORD (R. A.) (1971). - Purification and characterization of milk protease. Journal of Dairy Science, 54, 763-766.

DRIESSEN (F. M.) (1981). - Enzymatische eiwitafbraak in kort-hoog gesterilizeerde melkprodukten. 1. Produktie van proteïnasen door bakteriën tijdens hun groei in melk. Voedingsmiddelentechnologie, 14, 18-20.

DRIESSEN (F. M.), VAN DER WAALS (C. B.) (1978), - Inactivation of native milk proteïnase by heat treatment. Netherlands Milk and Dairy Journal, 32, 245-251.

E.E.G. (1981). - Onderzoek op wei in magere melkpoeder bestemd voor opslag d.m.v.bepaling van de hoeveelheid vrij sialinezuur. Publikatieblad van de Europese Gemeenschappen, L 213, 24, 3-6.

FluCKIGER (E.) (1976). - What happens in deep-refrigerated milk ? Nordeuropaeisk Mejeri-Tidsskrift, 7, 228-236.

Gebre-Egziabher (A.), Humbert (E. S.), Blankenagel (G.) (1980). - Hydrolysis of milk proteins by microbial enzymes. Journal of Food Protection, 43, 709-713.

GED (J.), Alais (C.) (1976). - Activité protéasique persistant dans les laits stérilisés. Le Lait, 56, 645-651.

Jolles (P.), Alats (C.), Jolles (J.) (1961). - Etude camparée des caséinoglycopeptides formés par action de la présure sur les caséines de vache, de brebis et de chèvre. I. Etude de la partie peptidique. Biochemica et Biophysica Acta, 51, 309-314.

KeLLER (H.) (1968). - Verzuche zur Anwendund der Rezazurinprobe bei trefgekühlt gelagerter Anlieferungsmilch. Deutsche Molkerei-Zeitung, 89, 483-485.

LAW (B. A.) (1979). - Review of the progress of Dairy Science: Enzymes of psychrotrophic bacteria and their effects on milk and milk products. Journal of Dairy Research, 46, 573-588.

LAW (B. A.), Andrews (A. T.), Sharpe (M. E.) (1977). - Gelation of UHT-sterilized milk by proteases from a strain of Pseudomonas fluorescens isolated from raw milk. Journal of Dairy Research, 44, 145-148. 
Marrier (J. R.), Tessier (H.), Dyson (R.) (1963). - Sialic acid as an index of the K-casein content of bovine skim milk. Journal of Dairy Science, 46, 373-379.

MotTaR (J.) (1981). - Heat resistant enzymes in UHT milk and their influence on sensoric changes during uncooled storage. Milchwissenchaft, 36, 87-91.

MotTAR (J.) (1983). - Karakterizering en eigenschappen van ultrahoog verhitte melk. Thesis, Rijksuniversiteit Gent.

Mottar (J.), Waes (G.), Moermans (R.), Naudts (M.) (1979). - Sensoric changes in UHT-milk during uncooled storage. Milchwissenschaft, 34, 257-262.

Nitschmann (H.), BeEby (R.) (1960). - Das Lab und Seine Wirkung auf das Casein der Milch. XIV. Aminosaurezusammensetzung des aus K-casein dur Lab in Freiheit gesetzen glycomacropeptids. Chimia, 14, 318-323.

Noomen (A.) (1975). - Proteolytic activity of milk protease in raw and pasteurized milk. Netherlands Milk and Dairy Journal, 29, 153-156.

RichaRdSON (B. C.), NEWSTEAD (D. F.) (1979). - Effect of heat-stable proteases on storage-life of UHT milk. New Zealand Journal of Dairy Science and Techno$\log y, 14,273-279$.

Richardson (B. C.), Te WhatTI (T.E.) (1978). - Partial characterization of heat stable extracellular proteases of some psychrotrophic bacteria form raw milk. New Zealand Journal of Dairy Science and Technology, 13, 172-176.

SNoeren (T. H. M.), Evers (P. H.) (1978). - Eiwitafbraak in stromend gesterilizeerde melk. Zuivelzicht, 70, 144-146.

Snoeren (T. H. M.), Van Riel (J. A. M.), Both (P.) (1979). - Enkele eigenschappen van het uit UHT gesterilizeerde melk geisoleerde melkproteïnase. Voedingsmiddelentechnologie, 12, 26-28.

SPECK (M. L.) (1976). - Compendium of methods for microbial examination of foods. American Public Health Association, Washington.

Yamauchi (K.), Kaminogawa (S.) (1972). - Decomposition of milk proteins by milk protease. Agricultural and Biological Chemistry, 36, 249-253. 